Provided for non-commercial research and education use. Not for reproduction, distribution or commercial use.

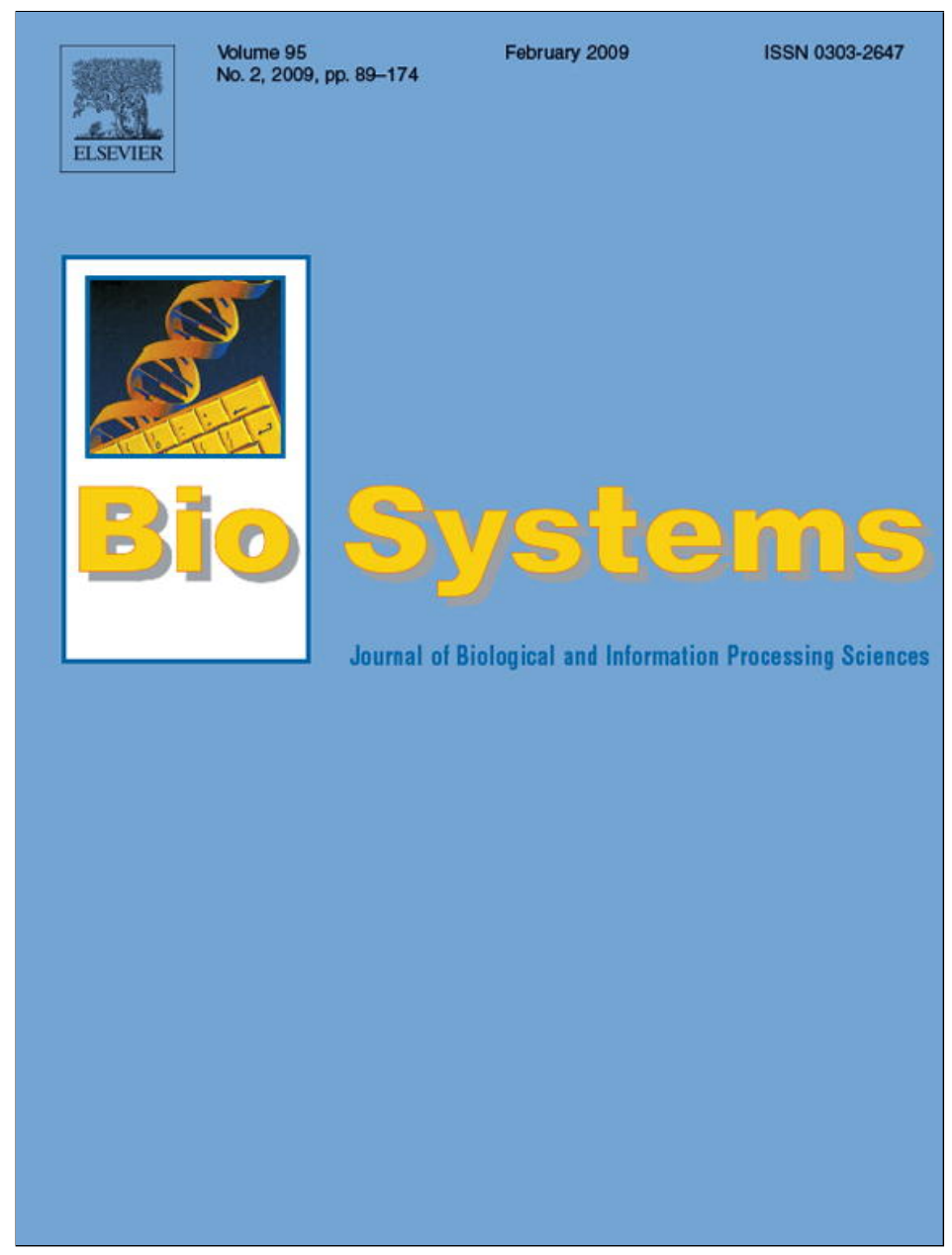

This article appeared in a journal published by Elsevier. The attached copy is furnished to the author for internal non-commercial research and education use, including for instruction at the authors institution and sharing with colleagues.

Other uses, including reproduction and distribution, or selling or licensing copies, or posting to personal, institutional or third party websites are prohibited.

In most cases authors are permitted to post their version of the article (e.g. in Word or Tex form) to their personal website or institutional repository. Authors requiring further information regarding Elsevier's archiving and manuscript policies are encouraged to visit:

http://www.elsevier.com/copyright 


\title{
A cultured human neural network operates a robotic actuator
}

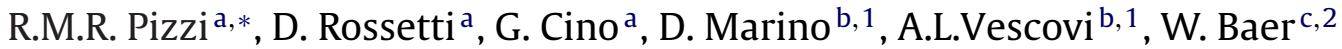 \\ a Living Networks Lab, Department of Information Technologies, University of Milan, Via Bramante 65, 26013 Crema (CR), Italy \\ b Stem Cell Research Institute, DIBIT S. Raffaele, Via Olgettina 58, 20132 Milano, Italy \\ c Department of Information Science, Naval Postgraduate School, 1 University Circle, Monterey, CA, USA
}

\section{A R T I C L E I N F O}

\section{Article history:}

Received 31 August 2007

Received in revised form

21 September 2008

Accepted 24 September 2008

\section{Keywords:}

MEA

Neurons

Stem cells

Bionics

Robot

Artificial neural networks

\begin{abstract}
A B S T R A C T
The development of bio-electronic prostheses, hybrid human-electronics devices and bionic robots has been the aim of many researchers. Although neurophysiologic processes have been widely investigated and bio-electronics has developed rapidly, the dynamics of a biological neuronal network that receive sensory inputs, store and control information is not yet understood. Toward this end, we have taken an interdisciplinary approach to study the learning and response of biological neural networks to complex stimulation patterns. This paper describes the design, execution, and results of several experiments performed in order to investigate the behavior of complex interconnected structures found in biological neural networks.

The experimental design consisted of biological human neurons stimulated by parallel signal patterns intended to simulate complex perceptions. The response patterns were analyzed with an innovative artificial neural network (ANN), called ITSOM (Inductive Tracing Self Organizing Map). This system allowed us to decode the complex neural responses from a mixture of different stimulations and learned memory patterns inherent in the cell colonies. In the experiment described in this work, neurons derived from human neural stem cells were connected to a robotic actuator through the ANN analyzer to demonstrate our ability to produce useful control from simulated perceptions stimulating the cells.

Preliminary results showed that in vitro human neuron colonies can learn to reply selectively to different stimulation patterns and that response signals can effectively be decoded to operate a minirobot. Lastly the fascinating performance of the hybrid system is evaluated quantitatively and potential future work is discussed.
\end{abstract}

(C) 2008 Elsevier Ireland Ltd. All rights reserved.

\section{Introduction}

In order to further the development of prosthetic biological interface through the expanded understanding of neurological signals we are perusing the in vitro investigation of biological networks based upon the assumption that such networks process complex spacio-temporal signal patterns as total integrated structures rather than serial neural pulse trains. A review of prior work, summarized in the following sections, showed us that we have little or no a priori knowledge of the neural processing or information content of the electronic activity observed in neural cell colonies. We have therefore connected biological neural network to an electronic artificial neural network (ANN) as two matched black boxes. Our goal was to show how the self-organizing properties of the

\footnotetext{
* Corresponding author. Tel.: +3902503 30072; fax: +39 0250330010 .

E-mail addresses: pizzi@dti.unimi.it (R.M.R. Pizzi), baer@nps.edu (W. Baer).

1 Tel.: +3902 26434952 .

2 Tel.: +1 8316562209 .
}

0303-2647/\$ - see front matter @ 2008 Elsevier Ireland Ltd. All rights reserved. doi:10.1016/j.biosystems.2008.09.006 latter can be used to adapt and extract information from the former. Our success suggests such a combination can be engineered into the architecture prosthetic interfaces and our knowledge of the electronic network, though complex, is tractable enough to provide insight into the workings of its complementary biological partner. After a brief summary of related work, we will present our experimental configuration, results, and preliminary insights in the following sections.

\subsection{Background}

An interest in developing a direct interface between electronics and neural cells began in the early 1990s with work by Fromherz et al. (1991), Fromherz et al. (1993), Fromherz and Schaden (1994), Weis et al. (1996), Jenkner and Fromherz (1997), and Schatzthauer and Fromherz (1998)). After these pioneering experiments, many laboratories investigated this field with the aim of developing future bio-electronic prostheses, hybrid human-electronics devices and bionic robots. Advances in neurophysiology were made by experiments with multi-electrode stimulation of animal neurons 
(Akin et al., 1994; Wilson et al., 1994; Bove et al., 1996; Canepari et al., 1997; Borkholder et al., 1997; Maher et al., 1999; Jimbo and Robinson, 2000; Egert et al., 2002). Using chaos theory, the Georgia Tech group (Schiff et al., 1994; Lindner and Ditto, 1996; Garcia et al., 2003) managed the chaotic electrical responses of interacting neurons extrapolating correct answers to simple computations.

In 2005 the group at the Trieste's International School for Advanced Studies (Ruaro et al., 2005) investigated the possibility of using neurons on Micro Electrode Arrays (MEAs) as "neurocomputers" able to filter digital images. While the Fromherz's group focused on ever more highly evolved chip-neuron junctions (Jenkner et al., 2001; Zeck and Fromherz, 2001; Bels and Fromherz, 2002; Bonifazi and Fromherz, 2002; Fromherz, 2002), the first steps in developing hybrid creatures formed by animal neurons connected to robotic arms or virtual animals were undertaken (Chapin et al., 1999; Reger et al., 2000; Taylor et al., 2002). In 2003 the group at Duke University (Carmena et al., 2003) inserted 320 microelectrodes into a monkey brain and the animal learned how to move a robotic arm. Potter's group at Georgia Tech (Potter, 2001; DeMarse et al., 2001; Wagenaar et al., 2001; De Marse et al., 2002) created a hybrid creature made of rat cortex neurons and this creature was able to learn from the environment. More recently, several groups including ours have designed closed-loop studies with natural-artificial hybrid systems (hybrots-neurobots): Kositsky et al. (2003), Wagenaar and Potter (2004), Martinoia et al. (2004), Potter et al. (2005). In Bakkum et al. (2004), under the control of a neural network cultured on MEA, a Koala 6-wheeled rover approached another randomly operated robot to demonstrate a rendezvous maneuver. The hybrid system encoded/decoded information on the base of the size of the neural interspike interval (ISI).

Nonetheless, the dynamics of neuronal networks that receive sensory inputs, store memories and control movement and behavior is not fully understood. The current methods are based on the detection and analysis of spike trains (Rieke et al., 1997; Borst and Theunissen, 1999; Paninski et al., 2004; Pillow et al., 2005), with interesting but inconclusive results.

Our group used a different approach. We started with the assumption that the neural signal has richer information content then what is contained in spike trains alone. Therefore it could be important to analyze such signals in toto. Such analysis would have to consider not only the spike frequencies but also the wideranging frequency variations and amplitudes of the whole signal. To achieve our aim, we developed a hybrid (biological-electronic) system composed of a network of human neurons connected to an ANN, which was in turn connected to a minirobot. A training sequence of simulated perceptions, in form of electrical stimulations, was used to stimulate the biological network. The output of the biological neurons was input of the ANN. This device was able to self-organize and classify the signals, by using the time series of the whole neural signal. As we will describe below, this allowed our hybrid electronic-neuronal system to learn simulated perceptions and to react correctly to an ensuing presentation of learned patterns.

\subsection{Past Experiments}

In the past experiments (Pizzi et al., 2004) cells were cultured on MEA connection sites and eight input channels taken from eight electrodes were connected to each other as a Hopfield network (Hopfield, 1984; Tank and Hopfield, 1989). In the Hopfield model, the output channels coincided with the input channels and therefore a short relaxation time (around $10 \mathrm{~ms}$ ) was introduced, after disconnecting the stimulation circuit, before the output signals were collected from the same electrodes. The biological neurons were stimulated by electrical pulses ranging from 1.25 to $25 \mathrm{~ms}$ using a variety of frequencies and voltages $(30-100 \mathrm{mV}$, suitable for neuron stimulation).

Our analyses, described in detail in Pizzi et al. (2007), highlighted the result that equal or similar stimulation patterns (i.e., many replications of the same pattern differing only by small variations attributed to noise) generated electrical signals with similar amplitude and shape, and different patterns generated welldiversified electrical signals. We also analyzed them qualitatively using Recurrence Quantification Analysis (RQA) (Takens, 1981; Zbilut and Webber, 1992; Kononov, 1996; Zbilut et al., 2002), evaluating the organization state of the biological networks before and after the training procedure. The result of this analysis indicated an increase of the response signal organization, i.e. an increase in their information content, as a consequence of the repeated stimulation. This lead us to the conclusion that repeated stimuli were modifying the network structure and both increasing and maintaining its information content suggesting a form of learning and memorization was occurring that could be measured and quantified.

\subsection{Present Experiment}

On the basis of these results we extended our research in order to improve our knowledge about neurophysiologic learning and memory functions and evaluate the feasibility of non-invasive neurological prostheses to substitute damaged brain functions. A new set of experiments was initiated with the aim of decoding the signals emitted from a biological neural network. We used a computer based artificial neural network, we call ITSOM, to provide a quantitative measure of learning occurring as the result of organized stimulation of a neuron culture. In the following sections we will describe the materials, electronic configuration, experiment operations, analysis, and results of these experiments. This is followed by a discussion of the significance of our findings and our plans for additional work we hope to conduct in the future.

\section{Materials}

\subsection{The MEAs}

Our system was based on the Microelectrode Array technology. A MEA is a glass Petri dish with small electrodes inserted. The cultured biological human neural cells adhere directly to these electrodes. Each electrode is connected by an isolated track to a pad suitable for an external connection. MEAs allow the recording of the simultaneous activity of many cells on different channels for an extended period without damaging the cultures.

Our Panasonic MEAs had 64 indium tin oxide (ITO)-platinum microelectrodes. The microelectrode size was $50 \mu \mathrm{m}$, the interpolar distance $150 \mu \mathrm{m}$. Their low, $10 \mathrm{k} \Omega$ impedance was critical to achieve a good signal-to-noise ratio necessary to investigate the dynamic behavior of a whole neural network.

\subsection{The Human Neural Cells}

In our experiments we used human fetal neural stem cells isolated in house in 1999 from the diencephalic/telencephalic area of human fetuses, spontaneously aborted at the tenth week of gestation (Vescovi et al., 1999), as a source of neurons.

The stem cells can be isolated and subcultured in vitro. They have the ability to divide and unlimitedly proliferate in the form of neurospheres by using the signaling of the fibroblast growth factor (FGF2) and the epidermal growth factor (EGF). After mechanical dissociation of the neurospheres, the resulting single stem cells can be plated on matrigel (an adhesion molecule derived by a mouse sarcoma, liquid state at $4{ }^{\circ} \mathrm{C}$, gel state after $20 \mathrm{~min}$ at $37^{\circ} \mathrm{C}$ ) and if suitably stimulated they can differentiate and become neurons, astrocytes and oligodendrocytes (McKay, 1997; Gritti et al., 1999, 2000, 2001; Galli et al., 2003). The neural cell maturation takes 18 days. Since stem cells are able to proliferate exponentially without requiring the use of multiple animal dissections and have the adaptability to grow in several different environments they are optimal candidates for possible future bionic implants.

The use of stem cells was suggested by the fact that this kind of cell has a discernible adaptability to adhere and differentiate on particular devices such as the MEAs. However it was important to verify that differentiated stem-derived cells would have appropriate electrophysiological properties required for our experi- 


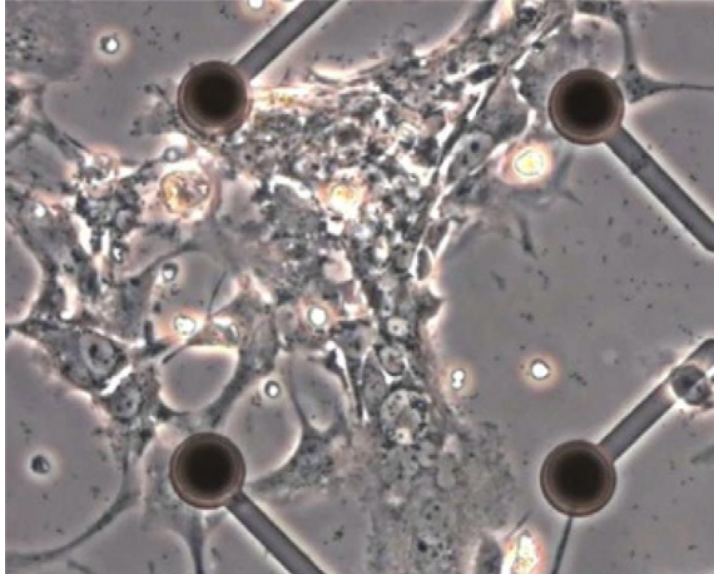

Fig. 1. Human fetal neural stem cells are able to mature on the MEA electrodes and become neural cells. Bright field, $10 \times$.

ments. The literature (Hiroki et al., 2000; Song et al., 2002) reported that adult neural stem cells isolated from a rat hippocampus, differentiated and stimulated by the brain-derived neurotrophic factor (BDNF), develop functional synapses in vitro. Human fetal neural stem cells with excitable properties were also observed in Gritti et al. (2000). In addition, the existence of in vitro synapses has also been demonstrated on the basis of morphological appearance (see Fig. 1).

Furthermore the increase of the current amplitude in the Na channels and the appearance of action potentials suggested that the channel functionalities grow gradually during neural cell maturation. We thus concluded that the existence of functional synapses in cultures of neural cells derived from adult neural stem cells has been experimentally proven and therefore were suitable candidates for our experiments.

\subsection{The Neural Cell Procedures}

Several technical procedures must be mentioned that are required to properly utilize the stem cell derived neurons and assure their proper functioning in the experiments.

MEAs must be thoroughly washed before use, because they are assembled using silicon, which could cause neurotoxicity. The electrodes must be perfectly clean to properly conduct the electrical signals. The washing protocol included $48 \mathrm{~h}$ of rinsing with distilled water and $70 \%$ ethanol, then an overnight exposure to UV light $(254 \mathrm{~nm})$ and a final sterile drying. The UV light treatment was performed on the MEAs without the presence of cells.

After the washing procedure, matrigel could be applied to the MEAs, however, we found that if cells were also added at this time the matrigel detached from the MEA after 2-3 days of culture and caused cell death (see Fig. 2).

This problem was solved by applying the matrigel on the MEA overnight before adding the cells. This protocol variation allowed good cell adhesion during the 25

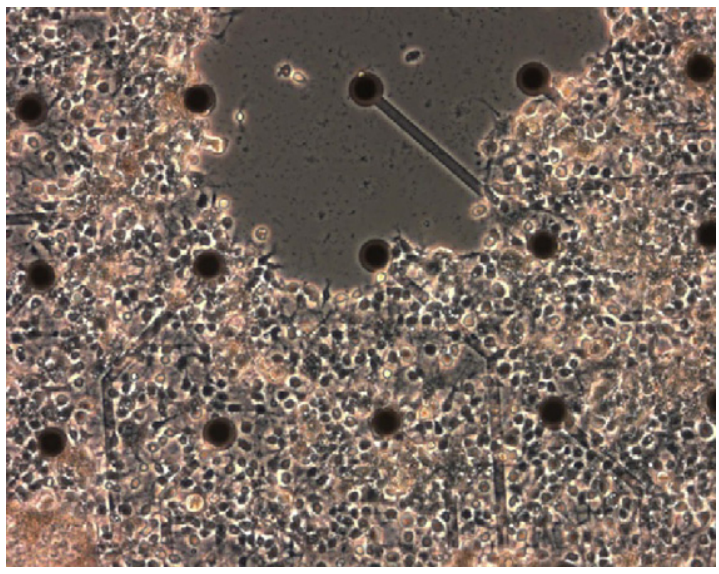

Fig. 2. Matrigel detachment leads to cell death. The MEA coating with matrigel proved to be a critical step because the gel was unable to adhere to the MEA for the total culturing time. Coating the MEA with matrigel overnight, however, solved the problem. Bright field, $5 \times$. days needed for cell maturation. Once the MEAs were coated, the neurospheres were mechanically dissociated and 50,000 single stem cells were plated and exposed to basic fibroblast growth factor (FGF2) and other neural growth factors such as brain derived neurotrophic factor (BDNF) for eighteen days before the learning experiment (Vescovi et al., 1993; Riaz et al., 2004). On the 19th day the MEAs were put inside a thermally controlled experimentation box at $37^{\circ} \mathrm{C}$.

To avoid electromagnetic noise the thermal resistors were carbon-based and DCsupplied. In order to ensure a good electromagnetical shielding, the plexiglass box was coated with a tight-knit $(1 \mathrm{~mm})$ brass mesh net. However, the thermo-controlled box did not insure a suitable atmosphere (oxygen $20 \%, \mathrm{CO}_{2} 5 \%$ ) for cell survival. Thus we exposed the cells to a made in-house Tyrode buffer $(134 \mathrm{mM} \mathrm{NaCl}, 3 \mathrm{mM} \mathrm{KCl}$, $1 \mathrm{mM} \mathrm{CaCl}_{2}, 1 \mathrm{mM} \mathrm{MgCl} 2,35 \mathrm{mM}$ glucose) which helps maintain the correct $\mathrm{pH}$ ( $\mathrm{pH}$ 7.4) and glucose levels. Addition of the Tyrode buffer to the cell medium resulted in two hours of cell survival, sufficient time for us to complete the experiments to be described in Section 3.

It is worth mentioning that in order to guarantee cells were vital throughout the experiment we treated cells with TTX (Tetrodotoxin) at the end of the experiment and performed one last recording. TTX is a potent neurotoxin extracted from the Tetraodontiformes family (which includes the pufferfish). It selectively blocks sodium ion transport through the membrane, thereby modifying excitability and inhibiting action potential.

As expected, after adding TTX to the cell culture, the voltage values were attenuated significantly until reaching a noise-level signal. This procedure demonstrates two important characteristics. First it shows that the recorded signals actually came from functional neuronal activity since such activity could be manipulated by neurotoxins, and second, it showed that the cells were active during the experiment, prior to the last recording. After all recording was completed, the cells were harvested from the MEAs and a viability assay was performed using Trypan Blue (a vital stain used to selectively color dead tissues or cells in blue). Though somewhat redundant this last test ensured that the cells, from which we were recording, had been alive during the experiment.

\subsection{The Hardware}

As described in previous works (Pizzi et al., 2004, 2007), our technique consisted of stimulating the cells with simulated perceptions in form of digital patterns consisting of organized bursts of multichannel electrical stimulations and then analyzing their response with an artificial neural network in order to command a robot. A block diagram of the hardware configuration used is shown in Fig. 3.

Stimulation patterns could be preset by a computer program that controlled a custom preamplifier circuit shown in Fig. 4. A custom device was required because when measuring low voltage signals from biological components care must be taken to avoid electromagnetic contamination from all external sources. To eliminate noise all the circuits were isolated and contained in a thick metal box connected to ground. The input and output signals were completely isolated by special Texas Instruments ISO124 chip that eliminates electromagnetic coupling.

In addition the output signals stimulating the MEA were uncoupled from the internal circuit by photocouplers. In this way the MEA electrodes were never in electrical contact with the outside. Four Li-ion batteries supplied a "clean" voltage, and the entire system was connected to ground.

The cells received electrical stimulations through eight shielded cables connected to the MEA and the same cables collected the electrical reactions of the cells to the simulated perceptions. The controller with the eight cables connected to the MEA inside the brass box is shown in Fig. 5 .

After amplification, the signals generated by the cells were acquired by a NI6052E National Instruments DAQ with the following specifications: $333 \mathrm{kS} / \mathrm{s}, 16$ bits, 16 analog inputs, 2 analog outputs, 8 digital I/O lines.

The digital data stream was then analyzed by the ANN software, which "learned" to classify the MEA response patterns into four possible commands and also generated classification confidence levels. When confidence levels exceeded preset thresholds the identified command could be sent to the robot. Thus in principle the robot would execute movements controlled by the response of the biological neural network to sensory stimulation. Our goal was less to demonstrate motor control than to further our understanding of complex signal environments in neural colonies. Further discussion of the signals, analysis and experimental results are provided in the next section.

\section{Experiment Protocol}

The basic experiment protocol consisted of a learning phase during which the neuron culture was stimulated with one of four patterns 10 times and the response used to tune the ITSOM ANN. The learning phase was followed by a test phase during which the culture was stimulated with a random sequence of the four patterns and its response analyzed by the ITSOM ANN. The number of correct stimulation pattern identifications is then used to quantify the 


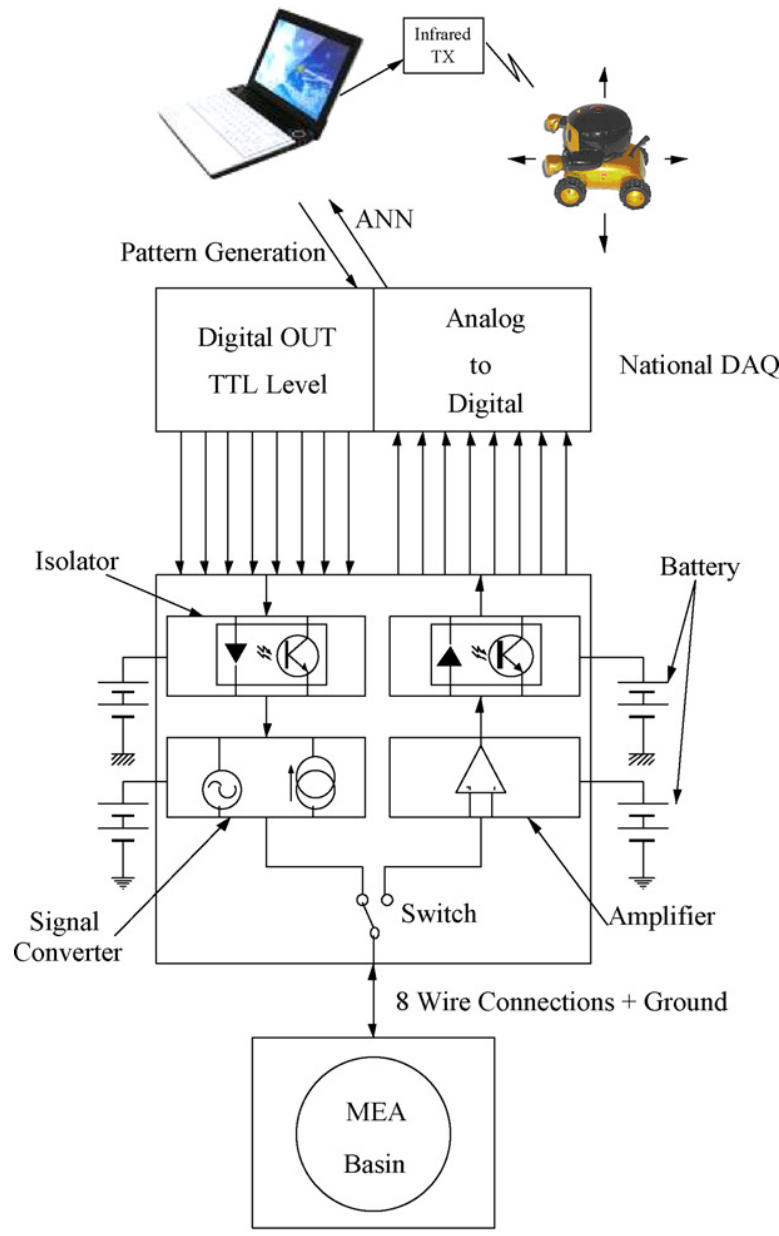

Fig. 3. Block diagram of the system.

amount of adaptation, i.e. learning in the culture. By repeating this basic experiment protocol with mature neurons, undifferentiated cells, and an untrained ITSOM ANN we hoped to show a significant statistical difference in the number of correct classifications and measure the learning capacity of the culture being tested.

The simulated perceptions took the form of four digital patterns. Each pattern consisted of a matrix of $8 \times 8$ bits shown as light and dark dots in Fig. 6. The rows represented the eight connected channels while the columns represented eight time intervals. A light colored bit meant the channel was activated a dark colored bit meant no electronic stimulation was sent on that channel at that time. An active stimulation consisted of a $433 \mathrm{~Hz}$ signal at $100 \mathrm{mV}$ of amplitude lasting $300 \mathrm{~ms}$. The stimulations were generated simultaneously on all the channels and yielded the whole pattern one row at a time. So for example the first and eighth MEA electrode on

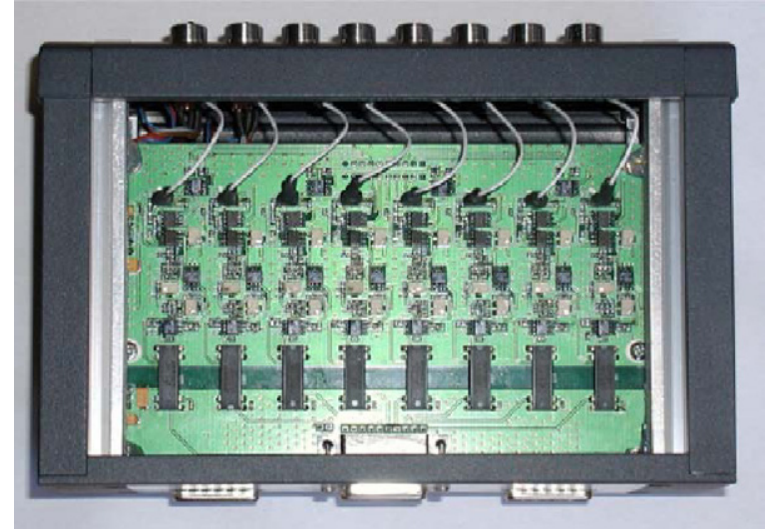

Fig. 4. The eight separated circuits of the custom preamplifier/controller.

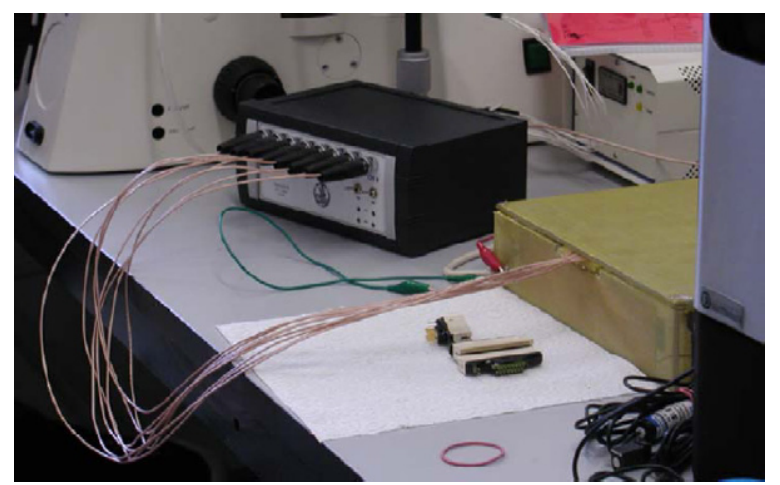

Fig. 5. The thermocontrolled Faraday cage containing the MEAs connected to the preamplifier/controller.

the Up arrow (left most) stimulation pattern would only be stimulated for $300 \mathrm{~ms}$ on the fourth and fifth time interval while the neurons attached to the fourth electrodes were stimulated for the entire $2.4 \mathrm{~s}$ required to complete the pattern.

It should be pointed out that the arrow-shaped bitmaps had no direct relationship with the electronics that operates the robot. They merely show a symbolic correspondence between pattern shape and robot direction. The arrow-shaped patterns were chosen because they are sufficiently complex and sufficiently distinct from one to another to support pattern classification in our experiment. Any four patterns with similar characteristics could be used.

During the training phase the patterns described above were used to stimulate the human neural cells for $2.4 \mathrm{~s}$ for each pattern. Each stimulation was followed by a $1 \mathrm{~s}$ pause and repeated 10 times. At the end of the tenth stimulation, the reactions of the human cells, collected after disconnecting the stimulation circuit and a $10 \mathrm{~ms}$ relaxation time, were recorded and sent to an artifi-
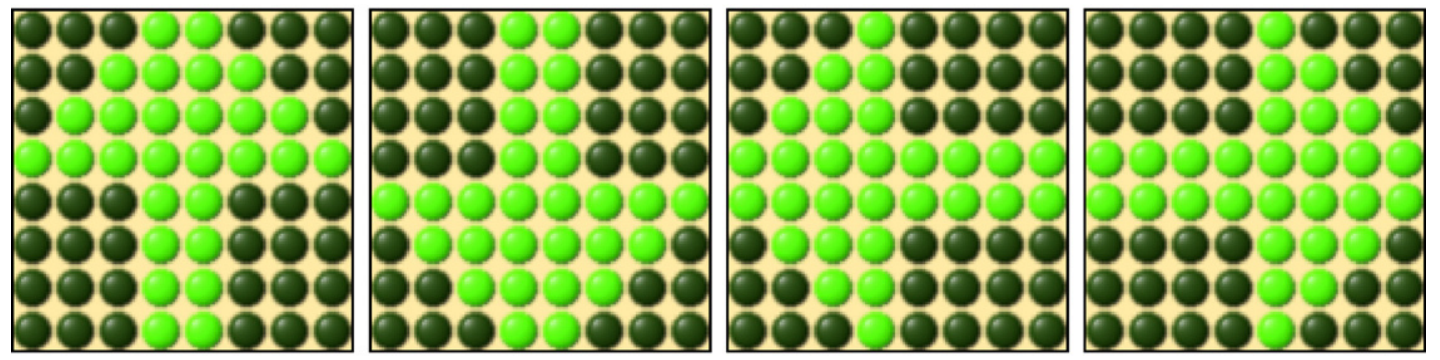

Fig. 6. The four arrow-shaped patterns: Forward, Backwards, Left, Right. 
cial neural network to be classified. From different off-line tests we observed that only the first $400 \mathrm{~ms}$ portion of the response signals contained information sufficient for correct classification. After this time interval the neural signal returns to random noise that has no further classification value and can be terminated.

Once the training sequence for one pattern was completed a new pattern was then selected and the sequence of ten pattern stimulations was repeated. Altogether 40 stimulation patterns were applied lasting approximately $100 \mathrm{~s}$. We developed a Labview graphical interface that allowed us to choose the commands both on an orderly basis (for training) and a random basis (for testing).

After the completion of the training phase the test phase was initiated. This consisted of a new sequence of 25 stimulations. Each of these individual stimulations had the shape of one of the four $2.4 \mathrm{~s}$ patterns described above. In contrast to the training phase the pattern sequence of the new individual stimulations was chosen randomly. Also in contrast to the training phase this time the electronics switched from stimulation to recording and the biological neuron response was presented to the ITSOM ANN after each of the 25 stimulations. The ANN thus performed 25 classification calculations outputting one of three results: either the ANN identified the correct stimulation pattern, or it identified the wrong stimulation pattern, or it did not converge to any identification in the time provided. Before presenting the results and discussing its significance we would like to describe the ITSOM ANN used to perform the classification in our experiment.

\subsection{The ITSOM Artificial Neural Network}

At first glance the response signals from neuron cultures look completely random and a robust mechanism is required to extract their inherent organization, if any, and classify such signals. To perform this function a novel ANN architecture called ITSOM (Inductive Tracing Self Organizing Map), was selected. A self-organizing architecture was necessary because we lacked a set of training examples. Furthermore the ITSOM network model is especially well suited for identifying structures in temporal series. The ITSOM has been shown to accurately show the organized structures of electrophysiological signals (Pizzi et al., 2002). The Self-Organizing Map (SOM) (Saarinen and Kohonen, 1985; Kohonen, 1990) features are well known. Also well known are its limits in classifying topologically entangled input structures.

To overcome these limits we developed the ITSOM architecture based on the following observation: even though the SOM winning weights vary at any given presentation epoch, their temporal sequence tends to repeat itself. The dynamical properties of the SOM have been investigated (Ritter and Schulten, 1986; Ritter and Schulten, 1988; Ermentrout, 1992) and show periodic oscillations and limit cycles. In particular the sequence of winning weights constitutes chaotic attractors that univocally characterize the input element that has determined them. Thus these sequences make it possible to finely classify the corresponding input value. A detailed description of the ITSOM's architecture is reported in (Pizzi, 1997; Pizzi et al., 2007).

After stimulation an electronic switch connects the MEA to the ITSOM, which begins to process the input signal. From off-line tests on recorded portions of these signals, we observed that only the first $400 \mathrm{~ms}$ contains sufficient information for correct classification. It should be emphasized that the crucial feature of the ITSOM is that the network does not need to converge because the cyclic configurations stabilize within a small number of epochs. The extremely low processing time makes this model very effective for real-time applications. Therefore the ITSOM processing cycle can be safely interrupted after few tenths of epochs and the winning sequences read out.
Once these sequences were available, an algorithm was needed to codify the obtained chaotic configurations of winning weights into a small set of outputs. To this end the cumulative scores related to each input were normalized following the distribution of the standardized variable $z$ given by

$z=\frac{x-\mu}{\sigma}$

where $\mu$ is the average of the scores on all the competitive layer weights and $\sigma$ is the root mean squared deviation. Once a threshold is fixed: $0<\tau \leq 1$, we have

$z=1$ when $z>\tau$,

$z=0 \quad$ when $z \leq \tau$.

In this way every winning configuration is represented by a binary number, or $z$-scores, with as the same number of bits as there are competitive layer weights. Due to the existence of a threshold, these $z$-scores coincide whenever the series of winning sequences are approximately similar. Then the task of comparing $z$-scores becomes straightforward and allows us to identify similar or identical inputs.

In our experiment the ITSOM must memorize the four states representing the movement of the robot by acquiring the signals generated by the cells during training. The network acquires such information by means of a matrix of floating point values $(10,000 \times 8$ for each directional pattern $)$.

We utilized a series of off-line trials to optimized the ITSOM for this task with the following parameters: 500 input neurons, 12 competitive layer neurons, Learning rate of 0.003 , Forgetting rate of 0.001 , and $\tau=0$. After finishing the training phase, the ITSOM knows the $z$-scores of the four patterns. During the testing phase we sent the neurons 25 stimulations, corresponding to one of the four patterns, in a random order. The ITSOM generates new $z$-scores and compares them to the ones stored after the training phase in order to produce the desired classification output.

\section{Results}

The basic experiment protocol was run on non-differentiated cells, mature neurons with the ITSOM not tuned and mature neurons with the ITSOM tuned as discussed in the last section. Three results types were recorded for each trial. When the one of the random test stimulation patterns was correctly identified by the ITSOM a Correct answer was noted. When a different pattern was identified it was considered a Wrong answer. When no pattern was identified it was marked as a Not Classified answer.

The behavior of the non-differentiated cells proved to be random. Analogous random results were obtained in a testing procedure carried out before the training phase. Experiments with mature neurons the following results were obtained.

A non-tuned ITSOM we collected 8 out of 25 Correct answers, 13 out of 25 Wrong answers, and 4 Not classified patterns. These results are graphically depicted in Fig. 7 . The $x$-axis represents the robot answers. The long bars describe the correctly classified patterns, the short bars the non-classified patterns, the absence of the bar indicates wrongly classified patterns.

With a tuned ITSOM using the above-mentioned parameters, we obtained the following results (Fig. 8): 15 out of 25 Correct answers, 7 out of 25 Wrong answers, and 3 Not classified patterns.

We can immediately observe that classification of the last samples was less effective. $85.7 \%$ of wrong answers happened in the last 9 samples. We believe this bias and the low number of samples obtained are due to the inadequate lifetime of the human neural stem cells that were in a less-than-optimal atmosphere and 


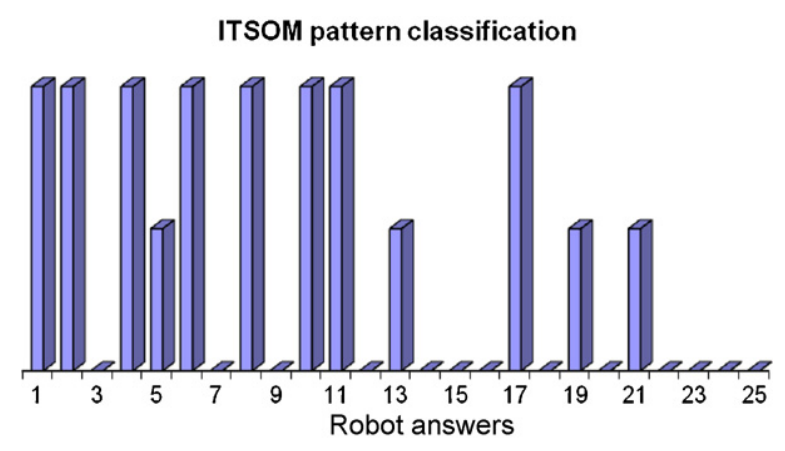

Fig. 7. Histogram of the classification performed by the non-tuned ITSOM.

ITSOM pattern classification after tuning

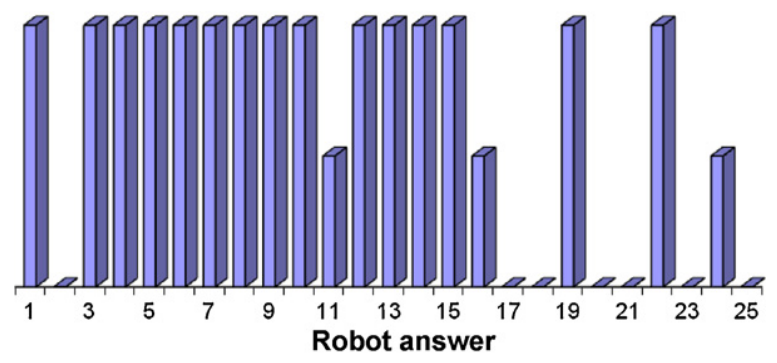

Fig. 8. Histogram of the classification performed by the ITSOM after tuning.

exposed to electrical stimulations. In fact, before the 25 random stimulations, the cells were subjected to 40 previous stimulations (10 for each command) needed for the training. We believe that after more than 60 electrical stimulations the cells might have been damaged. Alternatively the stimulation of a large number of random patterns may have erased the memory organization imposed during the training sessions.

Further analysis of the results is shown in Tables 1 and 2. Table 1 shows the classification percentage of the "F" and " $\mathrm{B}$ " patterns reach

Table 1

Robot performances.

\begin{tabular}{lccccc}
\hline Input & \multicolumn{2}{c}{ Directions } & & & \\
\cline { 2 - 5 } & Pattern F & Pattern B & Pattern L & Pattern R & \\
\hline Correct classification & 4 & 5 & 3 & 3 & 15 \\
Wrong classification & 1 & 0 & 3 & 3 & 7 \\
No classification & 0 & 1 & 1 & 1 & 3 \\
Yielded patterns & 5 & 6 & 7 & 7 & 25 \\
\% Classified & 100 & 83.33 & 85.71 & 85.71 & 88 \\
\% Correctly classified & 80 & 83.33 & 42.86 & 42.86 & 60 \\
\hline
\end{tabular}

Table 2

Classification percentage.

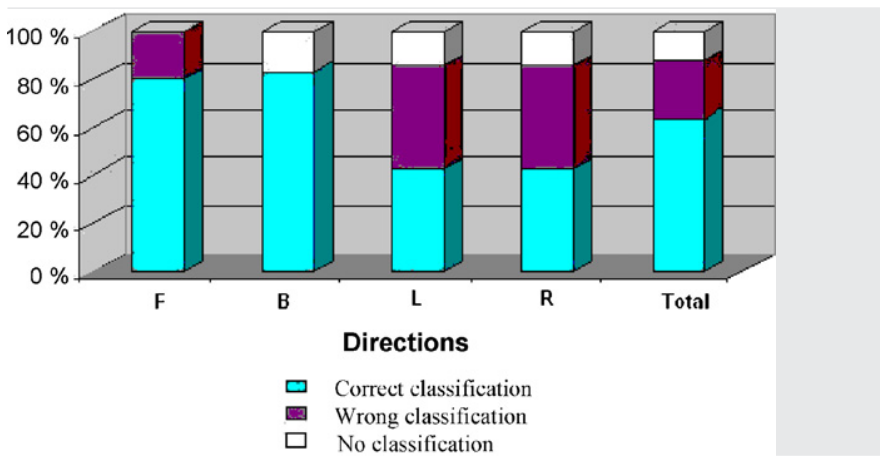

Table 3

Confusion matrixes of the model.

\begin{tabular}{|l|c|c|}
\hline \multicolumn{3}{|c|}{ Confusion matrix of a pattern « $P$ » } \\
\hline & P & Non-P \\
\hline P & TP & FN \\
\hline Non-P & FP & TN \\
\hline
\end{tabular}

\begin{tabular}{|c|c|c|}
\hline \multicolumn{3}{|c|}{ Confusion matrix pattern F } \\
\hline & F & Non-F \\
\hline F & 4 & 0 \\
\hline Non-F & 1 & 17 \\
\hline \hline \multicolumn{2}{|c|}{ Confusion matrix pattern L } \\
\hline \multicolumn{2}{|c|}{ L } & Non-L \\
\hline L & 3 & 1 \\
\hline Non-L & 3 & 15 \\
\hline
\end{tabular}

\begin{tabular}{|c|c|c|}
\hline \multicolumn{3}{|c|}{ Confusion matrix pattern $B$} \\
\hline & B & Non-B \\
\hline B & 5 & 6 \\
\hline Non-B & 0 & 11 \\
\hline \multicolumn{3}{|c|}{ Confusion matrix pattern $\mathbf{R}$} \\
\hline & $\mathbf{R}$ & Non-R \\
\hline $\mathbf{R}$ & 3 & 0 \\
\hline Non-R & 3 & 16 \\
\hline
\end{tabular}

Table 4

Total sensitivity and specificity.

\begin{tabular}{llllll}
\hline & Pattern F & Pattern B & Pattern L & Pattern R & Total \\
\hline Sensitivity & $100 \%$ & $45.45 \%$ & $75 \%$ & $100 \%$ & $80.11 \%$ \\
Specificity & $94.44 \%$ & $100 \%$ & $83.33 \%$ & $84.21 \%$ & $90.50 \%$ \\
\hline
\end{tabular}

high values ( $80 \%$ and $83.33 \%$ ). The $\mathrm{B}$ pattern is recognized with a $100 \%$ percentage. All the values are quite far from the random value $(25 \%)$. This is the value calculated from a simple one in four probability calculation. It should be pointed out that the network makes a choice among countless non-classifiable $z$-scores. This is due both to the ITSOM's effectiveness and the correct information content of the biological signals. This is also confirmed by the fact that $z$ score results for random non-differentiated cell trials show almost no correct answers.

In order to estimate the quality of this classification we calculated two-class confusion matrixes. Four significant parameters can be defined for each confusion matrix: False Positive (FP), False Negative (FN), True Positive (TP), True Negative (TN). Once we defined the sensitivity and specificity of a test by the following formulas, where TPs are the true positive classifications, TN the true negative, FN the false negative and FP the false positive:

Sensitivity $=\frac{\mathrm{TP}}{\mathrm{TP}+\mathrm{FN}} \times 100$
Specificity $=\frac{\mathrm{TN}}{\mathrm{TN}+\mathrm{FP}} \times 100$

for the four patterns we obtained the results described in Table 3.

Lastly in the summary (Table 4), the evaluation of the proposed model presents an accuracy of $80.11 \%$ and a precision of $90.50 \%$.

These results indicate the reasonably satisfactory effectiveness of our hybrid classifier.

\section{Discussion and Conclusions}

The aim of our research is twofold: to improve our knowledge of neurophysiologic learning and memory functions and evaluate the feasibility of biological computation or non-invasive 
neurological prostheses that can improve or substitute damaged brain functions. To achieve our goal we developed a hybrid (biological-electronic) system composed of a network of human neurons derived from human fetal neural stem cells connected to an ANN and a minirobot. ${ }^{3}$ Such a system allows us to analyze neural signal for information content that is richer than the content formed only by spike trains.

Despite the lack of a detailed physiological interpretation of the neural signals, the ANN worked as a black box and managed to decode the information hidden in the neural response using the whole signal samples. In fact the hybrid system, tested with 25 random patterns, obtained a correct classification of the four patterns with percentages of $80 \%, 83.33 \%, 42.86 \%$, and $42.86 \%$ respectively. The evaluation of the proposed model presents an accuracy of $80.11 \%$ and a precision of $90.50 \%$. Since trials with undifferentiated cells gave completely random results and random results were also obtained when the testing procedure was carried out before the training phase, we believe it is fair to conclude that in vitro learning and memory has been quantitatively measured and a methodology for such measurements established with our experiments.

Furthermore we observed that correct classification depends heavily on the degree of vitality and maturity of the neural cells, and consequently on their ability to self-organize and memorize patterns. We have also seen that a well-tuned ANN provides increased performance than a non-tuned one, but the ANN alone cannot classify the neural signals unless they originate from trained biological cells.

The wrong classifications of some patterns may be due to several factors:

- an attenuated viability of the cells

Although tests described in Section 2.3 do largely eliminate the possibility of cell death or even cell fatigue this possibility must be considered and tests improved to determine the exact viability of connecting cells during experimentation.

- a suboptimal ITSOM tuning

We already showed that the improvement of the ITSOM tuning changes dramatically the system performances. Thus a further work on tuning may lead to even better classification.

- an intrinsic limit of its classification algorithm

Other algorithms are under study, in particular a fuzzy algorithm that substitutes the $z$-score method in order to better identify similar chaotic attractors in signals.

- suboptimal electrical stimulation parameters

The choice of physiological stimulation parameters is a well-known issue that is constantly under study in all the neurophysiology research groups.

- forgetting effect in fatigued cells

If organized stimulation can train cells random stimulation may dilute the training effect. The extent to which learning is long term, or effected by fatigue, must be investigated if such training is to be used for control device interfaces.

In future experiments we hope to improve the stimulation parameters by evaluating different waveforms and polarization/depolarization times, amplitudes, frequencies and length of stimulation.

We will also study the behavior of different cell cultures submitted to repeated differential sampling of the training and testing

\footnotetext{
${ }^{3}$ In honor of our town, Crema, we called our hybrid creature "Cremino". We consider "Cremino" the first hybrid creature endowed with a small human "brain". An excerpt of its movements during the described experiment can be found in Pizzi (2006).
}

data, in order to compare and evaluate their resulting signals. A better tuning of the ANN is ongoing and a new algorithm that substitutes the $z$-score procedure is also being studied.

The new algorithm searches for a set of maximally winning neurons within a restricted set of epochs: in fact, the ITSOM shows a global preference towards a series of neurons specific to each pattern. During an off-line experiment the new procedure achieved better performances in the classification of the proposed patterns. The satisfactory results obtained up to now are encouraging us to improve the complexity of our hybrid system.

A new hardware controller handling 64 electrodes has been developed and tested that will enable design of much more complex patterns and a sharp increase of the number of connections. Using the new controller we will develop a system that can receive real perceptions from suitable sensors and react autonomously to environmental stimulations, exhibiting closed-loop performances. We will start adopting a controlled environment and low-resolution sensors.

We hope that new progress will lead us step by step to a better understanding of the neural code and the learning process, that in the future could be applied to technological devices such as brain implants and bionic systems.

\section{Acknowledgements}

We are deeply indebted to Prof. G. Degli Antoni (Department of Information Technologies, University of Milan) for his valuable suggestions and encouragement, to Dr. D. Carne, Dr. A. Redolfi and Dr. R. Rossoni (Department of Biomolecular Sciences and Biotechnologies, University of Milan) for their substantial contribution.

\section{References}

Akin, T., Najafi, K., Smoke, R.H., Bradley, R.M., 1994. A micromachined silicon electrode for nerve regeneration applications. IEEE Trans. Biomed. Eng. 41, 305313.

Bakkum, D.J., Shkolnik, A.C., Ben-Ary, G., Gamblen, P., DeMarse, T.B., Potter, S.M., 2004. Removing some 'A' from AI: embodied cultured networks. In: Iida, F., Pfeifer, R., Steels, L., Kuniyoshi, Y. (Eds.), Embodied Artificial Intelligence, vol. 3139. Springer, New York, pp. 130-145.

Bels, B., Fromherz, P., 2002. Transistor array with an organotypic brain slice: field potential records and synaptic currents. Eur. J. Neurosci. 15, 999-1005.

Bonifazi, P., Fromherz, P., 2002. Silicon chip for electronic communication between nerve cells by non-invasive interfacing and analog-digital processing. Adv. Mater., 17.

Borkholder, D.A., Bao, J., Maluf, N.I., Perl, E.R., Kovacs, G.T., 1997. Microelectrode arrays for stimulation of neural slice preparations. J. Neurosci. Methods 7, 61-66.

Borst, A., Theunissen, F.E., 1999. Information theory and neural coding:. Natre Neurosci. 2 (11), 947-957.

Bove, M., Martinoia, S., Grattarola, M., Ricci, D., 1996. The neuron-transistor junction: linking equivalent electric circuit models to microscopic descriptions. Thin Solid Films 285, 772-775.

Canepari, M., Bove, M., Mueda, E., Cappello, M., Kawana, A., 1997. Experimental analysis of neural dynamics in cultured cortical networks and transitions between different patterns of activity. Biol. Cybernet. 77, 153-162.

Carmena, J.M., Lebedev, M.A., Crist, R.E., O’Doherty, J.E., Santucci, D.M., Dimitrov, D.F., Patil, P.G., Henriquez, C.S., 2003. Learning to control a brain-machine interface for reaching and grasping by primates. M.A.L. PLoS 1, 193-208.

Chapin, J.K., Moxon, K.A., Markowitz, R.S., Nicolelis, M.A.L., 1999. Real time control of a robot arm using simultaneously recorded neurons in the motor cortex. Nature Neurosci. 2, 664-670.

De Marse, T.B., Wagenaar, D.A., Potter, S.M., 2002. The Neurally-controlled Artificial Animal: A Neural Computer Interface Between Cultured Neural Networks and a Robotic Body. SFN 2002, Orlando, Florida.

DeMarse, T.B., Wagenaar, D.A., Blau, A.W., Potter, S.M., 2001. The neurally controlled animat: biological brains acting with simulated bodies. Autonomous Robots 11, 305-310.

Egert, U., Schlosshauer, B., Fennrich, S., Nisch, W., Fejtl, M., Knott, T., Müller, T., Hammerle, H., 2002. A novel organotypic long-term culture of the rat hippocampus on substrate-integrated microelectrode arrays. Brain Resour. Protoc. 2, 229-242.

Ermentrout, B., 1992. Complex dynamics in WTA neural networks with slow inhibition. Neural Networks 5, 415-431.

Fromherz, P., Offenhäusser, A., Vetter, T., Weis, J., 1991. A neuron-silicon junction: a Retzius-cell of the leech on an insulated-gate field-effect transistor. Science 252, 1290-1293. 
Fromherz, P., Muller, C.O., Weis, R., 1993. Neuron-transistor: electrical transfer function measured by the Patch-Clamp technique. Phys. Rev. Lett. 71, 4079-4082.

Fromherz, P., Schaden, H., 1994. Defined neuronal arborisations by guided outgrowth of leech neurons in culture. Eur. J. Neurosci., 6.

Fromherz, P., 2002. Electrical interfacing of nerve cells and semiconductor chips. Chem. Phys. Chem. 3, 276-284.

Galli, R., Gritti, A., Bonfanti, L., Vescovi, A.L., 2003. Neural stem cells: an overview. Circul. Res. 92 (6), 598-608.

Garcia, P.S., Calabrese, R.L., DeWeerth, S.P., Ditto, W., 2003. Simple arithmetic with firing rate encoding in leech neurons: simulation and experiment. In: Proceedings of the XXVI Australian Computer Science Conference, Adelaide, vol. 16, pp. 55-60.

Gritti, A., Frolichsthal-Schoeller, P., Galli, R., Parati, E.A., Cova, L., Pagano, S.F., Bjornson, C.R., Vescovi, A., 1999. Epidermal and fibroblast growth factors behave as mitogenic regulators for a single multipotent stem cell-like population from the subventricular region of the adult mouse forebrain. J. Neurosci. 19 (9) 3287-3297.

Gritti, A., Rosati, B., Lecchi, M., Vescovi, A.L., Wanke, E., 2000. Excitable properties in astrocytes derived from human embryonic CNS stem cells. Eur. J. Neurosci. 12 (10), 3549-3559.

Gritti, A., Galli, R., Vescovi, A.L., 2001. In: Federoff(Ed.), Culture of Stem Cell of Central Nervous System. Humana Press III, pp. 173-197.

Hiroki, T., Jun, T., Akira, M., Konomi, K., Nobuo, H., 2000. Neurons generated from adult rat hippocampal stem cells form functional glutamatergic and GABAergic synapses in vitro. Exp. Neurol. 165 (1), 66-76.

Hopfield, J.J., 1984. Neural networks and physical systems with emergent collective computational abilities. Proc. Natl. Acad. Sci. U.S.A., 81

Jenkner, M., Fromherz, P., 1997. Bistability of membrane conductance in cell adhesion observed in a neuron transistor. Phys. Rev. Lett. 79, 4705-4708.

Jenkner, M., Muller, B., Fromherz, P., 2001. Interfacing a silicon chip to pairs of snail neurons connected by electrical synapses. Biol. Cybernet. 84, 239-249.

Jimbo, Y., Robinson, H.P.C., 2000. Propagation of spontaneous synchronized activity in cortical slice cultures recorded by planar electrode arrays. Bioelectrochemistry $5,107-115$.

Kohonen, T., 1990. Self-Organisation and Association Memory. Springer-Verlag.

Kononov, E., 1996. Available from: http://www.myjavaserver.com $/ \sim$ nonlinear/ vra/download.html.

Kositsky, M., Karniel, A., Alford, S., Fleming, K.M., Mussa Ivaldi, F.A., 2003. Dynamical dimension of a hybrid neurorobotic system. Trans. Neural Syst. Rehabil. Eng. 11, 155-159.

Lindner, J.F., Ditto, W., 1996. Exploring the nonlinear dynamics of a physiologically viable model neuron. AIP Conf. Proc. 1, 375-385.

Maher, M.P., Pine, J., Wright, J., Tai, Y.C., 1999. The neurochip: a new ultielectrode device for stimulating and recording from cultured neurons. Neurosci. Methods $87,45-56$.

Martinoia, S., Sanguineti, V., Cozzi, L., Berdondini, L., van Pelt, J., Tomas, J., Le Masson, G., Davide, F., 2004. Towards an embodied in vitro electrophysiology: the neurobit project. Neurocomputing 58-60, 1065-1072.

McKay, R.D.G., 1997. Stem cells in the central nervous system. Science 276, 66-71.

Paninski, L., Fellows, M., Hatsopoulos, N., Donoghue, J., 2004. Spatiotemporal tuning properties for hand position and velocity in motor cortical neurons. J. Neurophysiol. 91, 515-532.

Pillow, J.W., Paninski, L., Uzzell, V.J., Simoncelli, E.P., Chichilnisky, E.J., 2005. Predic tion and decoding of retinal ganglion cell responses with a probabilistic spiking model. J. Neurosci. 25, 11003-11013.

Pizzi, R., Fantasia, A., Gelain, F., Rossetti, D., Vescovi, A., 2004. Behavior of living human neural networks on microelectrode array support. In: Proceedings Nanotechnology Conference and Trade Show, Boston.

Pizzi, R., 1997. Theory of Dynamical Neural Systems with application to telecommunications. PhD Dissertation, University of Pavia.

Pizzi, R., de Curtis, M., Dickson, C., 2002. Evidence of chaotic attractors in cortical fast oscillations tested by an artificial neural network. In: Kacprzyk, J. (Ed.), Advances in Soft Computing. Physica Verlag.

Pizzi, R., 2006. Available from: <http://www.dti.unimi.it/ pizzi/research.html>.

Pizzi, R., Rossetti, D., Cino, G., Gelain, F., Vescovi, A., 2007. Learning in human neural networks on microelectrode arrays. Biosyst. J. 88(1-2), 1-15.
Potter, S.M., 2001. Distributed processing in cultured neuronal networks. In: Nicolelis (Ed.), Progress in Brain Research, M.A.L. Elsevier Science.

Potter, S.M., Wagenaar, D.A., DeMarse, T.B., 2005. Closing the loop: Stimulation Feeback Systems for embodied MEA cultures. In: Taketani, M., Baundry, M. (Eds.), Advances in Network Electrophysiology Using Multi-Electrode Arrays. Springer, New York.

Reger, B., Fleming, K.M., Sanguineti, V., Simon Alford, S., Mussa-Ivaldi, F.A., 2000 Connecting brains to robots: an artificial body for studying the computational properties of neural tissues. Artif. Life 6, 307-324.

Riaz, S.S., Theofilopoulos, S., Jauniaux, E., Stern, G.M., Bradford, H.F., 2004. The differentiation potential of human foetal neuronal progenitor cells in vitro. Brain Res. Dev. Brain Res. 153 (1), 39-51.

Rieke, F., Warland, D., de Ruyter van Steveninck, R., Bialek, W., 1997. Spikes: Exploring the Neural Code. MIT Press.

Ritter, H., Schulten, K., 1986. On the stationary state of Kohonen's self-organizing sensory mapping. Biol. Cybernet. 54, 99-106.

Ritter, H., Schulten, K., 1988. Convergence properties of Kohonen's topology conserving maps: fluctuations, stability, and dimension selection. Biol. Cybernet. $60,59-71$.

Ruaro, M.E., Bonifazi, P., Torre, V., 2005. Toward the neurocomputer: image processing and pattern recognition with neuronal cultures. IEEE Trans. Biomed. Eng. 3.

Saarinen, J., Kohonen, T., 1985. Self-organized formation of colour maps in a model cortex. Perception 14 (6), 711-719.

Schatzthauer, R., Fromherz, P., 1998. Neuron-silicon junction with voltage gated ionic currents. Eur. J. Neurosci. 10, 1956-1962.

Schiff, S.J., Jerger, K., Duong, D.H., Chang, T., Spano, M.L., Ditto, W., 1994. Controlling chaos in the brain. Nature 8,25 .

Song, H., Stevens, C.F., Gage, F.H., 2002. Neural stem cells from adult hippocampus develop essential properties of functional CNS neurons. Nature Neurosci. 5, 5.

Takens, F., 1981. Detecting strange attractors in turbulence. In: Rand, D.A., Young, L.S. (Eds.), Dynamical Systems and Turbulence - Lecture Notes in Mathematics, vol. 898. Springer-Verlag.

Tank, D.W., Hopfield, J.J., 1989. Neural Architecture and Biophysics for Sequence Recognition in Neural Models of Plasticity. Academic Press.

Taylor, D.M., Tillery, S.I.H., Schwarz, A.B., 2002. Direct cortical control of 3D neuroprosthetic devices. Science 296 (5574), 1829-1832.

Vescovi, A.L., Reynolds, B.A., Fraser, D.D., Weiss, S., 1993. bFGF regulate the proliferative fate of unipotent (neuronal) and bipotent (neuronal/atroglial) EFGgenerated CNS progenitor cells. Neuron 11 (5), 951-966.

Vescovi, A.L., Parati, E.A., Gritti, A., Poulin, P., Ferrario, M., Wanke, E., FrölichsthalSchoeller, P., Cova, L., Arcellana-Panlilio, M., Colombo, A., Galli, R., 1999. Isolation and cloning of multipotential stem cells from the embryonic human CNS and establishment of transplantable human neural stem cell lines by epigenetic stimulation. Exp. Neurol. 156, 71-83.

Wagenaar, D.A., Potter, S.M., 2004. A versatile all-channel stimulator for electrode arrays, with realtime control. J. Neural Eng. 1, 39-45.

Wagenaar, D.A., DeMarse, T.B., Potter, S.M., 2001. A toolset for realtime analysis of network dynamics in dense cultures of cortical neurons. 7th JSNC, University of California at San Diego, La Jolla.

Weis, R., Müller, B., Fromherz, P., 1996. Neuron adhesion on silicon chip probed by an array of field-effect transistors. Phys. Rev. Lett. 76, 327-330.

Wilson, R.J. Breckenridge, L., Blackshaw, S.E., Connolly, P., Dow, J.A.T., Curtis, A.S.G. Wilkinson, C.D.W., 1994. Simultaneous multisite recordings and stimulation of single isolated leech neurons using planar extracellular electrode arrays. Neurosci. Methods 53, 101-110.

Zbilut, J.P., Webber, C.L., 1992. Embeddings and delays as derived from quantification of recurrent plots. Phys. Lett., 171.

Zbilut, J.P., Thomasson, N., Webber Jr., C.L., 2002. Recurrence quantification analysis as a tool for nonlinear exploration of nonstationary cardiac signals. Med. Eng. Phys. 24, 53-60.

Zeck, G., Fromherz, P., 2001. Noninvasive neuroelectronic interfacing with synaptically connected snail neurons on a semiconductor chip. Proc. Natl. Acad. Sci. 98 10457-10462. 